BULLETIN OF THE

AMERICAN MATHEMATICAL SOCIETY

Volume 78, Number 5, September 1972

\title{
ON THE SUMMATION OF CONJUGATE FOURIER INTEGRALS OF FUNCTIONS OF SEVERAL VARIABLES
}

\author{
BY T. WALSH
}

Communicated by François Treves, February 9, 1972

Let $K$ denote a homogeneous Calderón-Zygmund singular integral kernel $K(x)$ which is bounded and has mean value 0 on the unit sphere $S^{n-1}=\{x:|x|=1\}$ of $R^{n}$ and let $\hat{K}$ denote its principal valued Fourier transform (see [2]). In this note some simple propositions are stated which generalize results of V. L. Shapiro and others and can be proved in an elementary fashion. Suppose the Fourier transform of any integrable function $f$ is defined by $\hat{f}(x)=\int f(y) e^{-i x y} d y$. The truncated kernel $K_{\varepsilon}$ is defined by $K_{\varepsilon}(x)=K(x)$ if $|x| \geqq \varepsilon$ or $K_{\varepsilon}(x)=0$ otherwise. For any function $g$ not denoted by $K$ and $\varepsilon>0$ let $g_{\varepsilon}(x)=\varepsilon^{-n} g\left(\varepsilon^{-1} x\right), g^{\varepsilon}(x)=g(\varepsilon x)$.

Proposition 1. Suppose $K(x)=\Omega(x)|x|^{-n}$ where $\Omega$ is homogeneous of degree 0 , has mean value 0 on $S^{n-1}$ and its modulus of continuity defined by

$$
\omega(t)=\sup \{|\Omega(\xi+h)-\Omega(\xi)|:|\xi|=1,|h| \leqq t\}
$$

satisfies the Dini condition $\int_{0}^{1} \omega(t) d t / t<\infty$.

Furthermore suppose the integrable function $\varphi$ is such that

$$
\begin{aligned}
& \psi_{0}(x)=\int_{1 \leqq|x-y| \leqq|x| / 2} K(x-y) \varphi(y) d y, \\
& \psi_{1}(x)=\int_{|y| \geqq|x|} \varphi(y) d y, \\
& \psi_{2}(x)=\int_{|y| \leqq 1}|y|^{-n}|\varphi(x-y)-\varphi(x)| d y
\end{aligned}
$$

all satisfy

$$
\int_{0}^{\infty} \sup _{t \leqq|x| \leqq 2 t}\left|\psi_{j}(x)\right| t^{n-1} d t<\infty .
$$

Finally suppose $\hat{\varphi} \in L^{1}$. Then, for $f \in L^{1}$ and $\int \varphi(x) d x=m$,

$$
\lim _{\varepsilon \rightarrow 0}\left[(2 \pi)^{-n} \int \hat{f}(y) \hat{K}(y) e^{i x y} \hat{\varphi}(\varepsilon y) d y-m K_{\varepsilon} * f(x)\right]=0
$$

AMS 1970 subject classifications. Primary 42A40, 42A92, 44A25.

Copyright (C) American Mathematical Society 1972 
for every point $x$ in the Lebesgue set of $f$.

Proposition 1 can be shown to imply the following:

COROLlaRY. (5) holds at every point $x$ in the Lebesgue set of $f$ provided $K$ is as in Proposition 1, $\varphi, \hat{\varphi}$ are integrable, $\psi_{2}$ defined by (3) satisfies (4) and

(a) $\int_{0}^{\infty} \sup _{t \leqq|x| \leqq 2 t}|\varphi(x)|\left(1+\log ^{+} t\right) t^{n-1} d t<\infty$ or

(b) $\psi_{1}$ satisfies (4) and there is a decomposition of $R^{n}$ into a family of disjoint measurable sets $E_{j}$ of bounded diameters $R^{n}=\bigcup_{j=0}^{\infty} E_{j}$ such that

$$
\begin{aligned}
& \int_{E_{j}}|\varphi(x)| d x \leqq \psi_{3}\left(\delta_{j}\right)\left|E_{j}\right|, \\
& \left|\int_{E_{j}} \varphi(x) d x\right| \leqq \psi_{3}\left(\delta_{j}\right)\left(1+\log ^{+} \delta_{j}\right)^{-1}\left|E_{j}\right|,
\end{aligned}
$$

where $\psi_{3}$ satisfies $\int_{0}^{\infty} \sup _{t \leqq s \leqq 2 t} \psi_{3}(s) t^{n-1} d t<\infty, \delta_{j}$ is the distance of $E_{j}$ from 0 and $\left|E_{j}\right|$ denotes the Lebesgue measure of $E_{j}$.

Proposition 1 can be proved by means of the following fairly obvious lemma.

LEMMA. Suppose $\Phi$ is measurable and

$$
\int_{0}^{\infty} \sup _{t \leqq|x| \leqq 2 t}|\Phi(x)| t^{n-1} d t<\infty
$$

then

$$
\lim _{\varepsilon \rightarrow 0} \Phi_{\varepsilon} * f(x)=f(x) \int \Phi(x) d x
$$

for every point $x$ in the Lebesgue set of $f$.

Proof of Proposition 1. $\hat{\varphi} \in L^{1}$ implies

$$
\int \hat{f}(y) \hat{K}(y) e^{i x y} \hat{\varphi}(\varepsilon y) d y=(2 \pi)^{n}(\text { p.v. } K * \varphi)_{\varepsilon} * f(x)
$$

so that (5) amounts to $\lim _{\varepsilon \rightarrow 0} \Phi_{\varepsilon} * f(x)=0$ where $\Phi=$ p.v. $K * \varphi-m K_{1}$. Let

$$
\Phi_{0}=\text { p.v. }\left(K-K_{1}\right) * \varphi, \quad \Phi_{1}=K_{1} * \varphi-m K_{1} .
$$

By the above lemma it suffices to show that (8) is satisfied by $\Phi_{j}$ and $\int \Phi_{j}(x) d x=0$ for $j=0,1$. For $\Phi_{0}$ these assertions follow directly from (4) for $j=2$, the fact that $K$ has mean value 0 on $S^{n-1}$ and dominated convergence. 
As for $\Phi_{1}$ observe that $\Phi_{1}=\sum_{m=1}^{4} \Phi_{1 m}$ where

$$
\begin{aligned}
& \Phi_{11}(x)=\int_{|y|<|x| / 2}\left(K_{1}(x-y)-K_{1}(x)\right) \varphi(y) d y, \quad \Phi_{12}(x)=\psi_{0}(x), \\
& \Phi_{13}(x)=\int_{|y| \geqq|x| / 2} K_{1}(x-y) \varphi(y) d y, \quad \Phi_{14}(x)=-K_{1}(x) \psi_{1}(x / 2) .
\end{aligned}
$$

Hence for $|x|>2$,

$$
\begin{aligned}
& \sup _{t \leqq|x| \leqq 2 t}\left|\Phi_{11}(x)\right| \leqq C t^{-n} \int_{|y|<|x| / 2} \omega(|y| / t)|\varphi(y)| d y, \\
& \sup _{t \leqq|x| \leqq 2 t}\left|\Phi_{13}(x)\right| \\
& \quad \leqq C\|\Omega\|_{\infty}\left(t^{-n} \int_{t / 2 \leqq|y| \leqq 2 t}|\varphi(y)| d y+\int_{|y| \geqq 2 t}|\varphi(y)||y|^{-n} d y\right) .
\end{aligned}
$$

It follows that

$$
\int \sup _{t \leqq|x| \leqq 2 t}\left|\Phi_{1 m}(x)\right| t^{n-1} d t \leqq C\|\Omega\|_{\infty} \int|\varphi(x)| d x, \quad m=1,3 .
$$

Furthermore by (4) for $j=0,1, \int_{0}^{\infty} \sup _{t \leqq|x| \leqq 2 t}\left|\Phi_{1 m}(x)\right| t^{n-1} d t$ is finite for $m=2,4$.

To see that $\int \Phi_{1}(x) d x=0$ notice that if $\lambda$ is a continuously differentiable radial function of compact support which equals 1 in a neighborhood of 0 then, for $\Phi_{1}^{(\varepsilon)}=\left(K_{1} \lambda^{\varepsilon}\right) * \varphi-m K_{1} \lambda^{\varepsilon}$, $\sup _{\varepsilon<1}\left|\Phi_{1}^{(\varepsilon)}\right|$ is still integrable and $\int \Phi_{1}^{(\varepsilon)}(x) d x=0$ for any $\varepsilon>0$, hence by dominated convergence $\int \Phi_{1}(x) d x=0$.

Part (a) of the Corollary, for instance, implies Abel and Bochner-Riesz summability (above the critical index $(n-1) / 2)$ of "conjugate" Fourier transforms $\hat{R} \hat{f}$ of integrable functions $f$ at any point where the singular integral p.v. $K * f$ exists and which is in the Lebesgue set of $f$ (see [3], [4], [5], [6]). Part (b) could be used to prove the same assertion for $\hat{\varphi}(x)$ $=\left(1-|x|^{2}\right)_{+}^{(n-1) / 2}\left[\log \left(e /\left(1-|x|^{2}\right)\right)\right]^{-\alpha}$ where $\alpha>1$, in which case the Corollary may no longer apply.

The following result generalizes a result of Wheeden (see [7]) about Bochner-Riesz summability of conjugate Fourier transforms at the critical index.

Proposition 2. Suppose $K$ is as in Proposition $1, \varphi$ is locally integrable, $\hat{\varphi} \in L^{1}$ and there is a decomposition of $R^{n}$ into a family of disjoint measurable 
sets of bounded diameters $R^{n}=\bigcup_{j=0}^{\infty} E_{j}$ such that

$$
\begin{aligned}
& \int_{E_{j}}|\varphi(x)| d x \leqq B\left(1+\delta_{j}\right)^{-n}\left|E_{j}\right|, \\
& \left|\int_{E_{j}} \varphi(x) d x\right| \leqq B\left(1+\delta_{j}\right)^{-n}\left(1+\log ^{+} \delta_{j}\right)^{-1}\left|E_{j}\right|,
\end{aligned}
$$

where $\delta_{j}$ is the distance of $E_{j}$ from 0 ,

$$
\lim _{s \rightarrow \infty} \int_{|x|<s} \varphi(x) d x=m .
$$

Finally suppose for $0<\varepsilon \leqq 1$

$$
|x|^{n} \int_{|y|<\varepsilon}|y|^{-n}|\varphi(x+y)-\varphi(x)| d y \leqq B(\varepsilon)
$$

where $B(\varepsilon) \leqq B$ and $\lim _{\varepsilon \rightarrow 0} B(\varepsilon)=0$.

If $f \in L^{1}$ and $\int|f(x+y)-f(x)||y|^{-n} d y<\infty$ then (5) is valid.

Since p.v. $K * \varphi$ belongs to the space $C_{0}$ of continuous functions vanishing at $\infty$ and since $L^{1}$ is dense in the space $\mathscr{M}^{1}$ of (complex) Borel measures of finite total variation with respect to the weak topology of the pairing with $C_{0}$ the above results generalize to conjugate Fourier-Stieltjes transforms. Furthermore by Poisson's summation formula Proposition 1 and the Corollary extend to periodic functions.

\section{REFERENCES}

1. S. Bochner, Summation of multiple Fourier series by spherical means, Trans. Amer. Math. Soc. 40 (1936), 175-207.

2. A. P. Calderón and A. Zygmund, On the existence of certain singular integrals, Acta Math. 88 (1952), 85-139. MR 14, 637.

3. S. P. Philipp, V. L. Shapiro and W. H. Sills, The Abel summability of conjugate multiple Fourier-Stieltjes integrals, Pacific J. Math. 36 (1971), 231-238. MR 43 \#2425.

4. V. L. Shapiro, The conjugate Fourier-Stieltjes integral in the plane, Bull. Amer. Math. Soc. 65 (1959), 12-15. MR 20 \#7187.

5. - Topics in Fourier and geometric analysis, Mem. Amer. Math. Soc. No. 39 (1961), 100 pp. MR 26 \#5339.

6. - Fourier series in several variables, Bull. Amer. Math. Soc. 70 (1964), 48-93. MR 28 \#1448.

7. R. L. Wheeden, Hypersingular integrals and summability of Fourier integrals and series, Proc. Sympos. Pure Math., vol. 10, Amer. Math. Soc., Providence, R.I., 1967, 336-369. MR 39 \#3364.

8. K. Yosida, Functional analysis, 2nd ed., Die Grundlehren der math. Wissenschaften, Band 123, Springer-Verlag, New York, 1968. MR 39 \#741.

Department of Mathematics, University of Florida, Gainesville, Florida 32603 